\title{
Compact noninvasive electron bunch-length monitor
}

\author{
B. Roberts \\ Electrodynamic, 4909 Paseo Del Norte Ne Suite D, Albuquerque, New Mexico 87113, USA \\ R. R. Mammei and M. Poelker \\ Thomas Jefferson National Accelerator Facility, 12000 Jefferson Avenue, Newport News, Virginia 23606, USA \\ J. L. McCarter \\ Department of Physics, University of Virginia, Charlottesville, Virginia 22901, USA
}

(Received 6 August 2012; published 26 December 2012)

\begin{abstract}
A compact rf cavity was constructed that simultaneously resonates at many harmonic modes when excited by a repetitive bunched electron beam passing through its bore. The excitation of these modes provides a Fourier description of the temporal characteristics of the bunch train. The cavity was used to noninvasively characterize electron bunches produced from thin and thick GaAs photocathodes inside a DC high voltage photogun illuminated with 37 ps (full width half maximum, FWHM) laser pulses at repetition rates near $1500 \mathrm{MHz}$, at average beam current from 5 to $500 \mu \mathrm{A}$, and at beam energy from 75 to $195 \mathrm{keV}$. The cavity bunch-length monitor could detect electron bunches as short as 57 ps (FWHM) when connected directly to a sampling oscilloscope, and could clearly distinguish bunches with varying degrees of space-charge induced growth and with different tail signatures. Efforts are under way to detect shorter bunches by designing cavities with increased bandwidth. This demonstration lends credibility to the idea that these cavities could also be used for other applications, including bunching and shaping, when driven with external rf.
\end{abstract}

DOI: 10.1103/PhysRevSTAB.15.122802

PACS numbers: 29.27.- a

\section{INTRODUCTION}

It is extremely important to understand the temporal characteristics of electron beams at accelerator photoinjectors. Measuring and setting the electron bunch length is critical to the operation of the accelerator, and can ultimately determine if physics experiments at user-based accelerators can be successfully performed. Many parameters affect the temporal characteristics of electron bunches at the photoinjector including the drive laser optical pulse, the photogun bias voltage, beam current and bunch charge, the field strength of focusing magnets, and the phase and field strength of $\mathrm{rf}$ bunching and accelerating cavities. Optimizing these parameters can be a time consuming process, particularly during accelerator commissioning. Wall-current monitors [1] that detect the image charge of the bunch traveling along the metal beam pipe are noninvasive but these devices are not particularly fast, with minimum rise time detection of $\sim 200 \mathrm{ps}$. An rf deflecting cavity can be used to measure shorter bunches (few ps) but this is an invasive measurement [2]. It is very desirable to have a compact, real-time, noninvasive bunch-length monitor, particularly one that can detect bunches with

Published by the American Physical Society under the terms of the Creative Commons Attribution 3.0 License. Further distribution of this work must maintain attribution to the author(s) and the published article's title, journal citation, and DOI. duration $<100$ ps. Such a device could be used to accomplish a number of important tasks, including setting the rf amplitude of bunching cavities, monitoring rf phase stability, and detecting bunch-length growth due to space charge forces.

A periodically bunched electron beam can be described mathematically as a Fourier series expansion, expressed in compact trigonometric form:

$$
\begin{aligned}
I(t)= & a_{0}+a_{1} \cos \left(\omega_{o} t+\theta_{1}\right)+a_{2} \cos \left(2 \omega_{o} t+\theta_{2}\right) \\
& +a_{3} \cos \left(3 \omega_{o} t+\theta_{3}\right)+\cdots,
\end{aligned}
$$

where $I(t)$ describes the time-varying electron beam current, the constant term $a_{0}$ represents a DC offset, $\omega_{o}$ is the bunch repetition frequency, and successive terms represent contributions at integer multiples of $\omega_{o}$. The amplitude and phase terms $\left(a_{n}, \theta_{n}\right)$ influence the bunch length and shape.

$\mathrm{TM}_{0 \mathrm{~N} 0}$ modes are axially symmetric and have a field maximum on the cavity axis. The cavity bunch-length monitor described herein was designed to exclusively resonate at many harmonic TM modes, with the first mode, $\left(\mathrm{TM}_{010}\right)$ the same as the bunch frequency, and higher order modes $\left(\mathrm{TM}_{020}, \mathrm{TM}_{030}\right.$, etc.) corresponding to harmonic frequencies of the Fourier series expansion. An ideal bunch-length monitor would be capable of resonating at harmonic frequencies beyond the harmonic content of the detected electron bunch, and with uniform phase and detection sensitivity at each mode. 


\section{EXPERIMENT}

\section{A. Cavity design and construction}

The cavity bunch-length monitor relies on three design criteria. First, the cavity design excludes TE modes. This was accomplished by noting that TE modes oscillate at frequencies $\geq c / 2 h$, where $h$ is the cavity length along the beam's direction of motion. A short, pancake-shaped cavity with length of a few millimeters readily excludes TE modes with frequencies below many tens of GHz. Second, radial slits were cut into the cavity walls to remove unwanted $\mathrm{TM}$ modes. The $\mathrm{TM}_{0 \mathrm{~N} 0}$ modes have purely radial wall currents and are unaffected by these slits while the $\mathrm{TM}_{\mathrm{MNP}}$ modes with azimuthal mode numbers $(\mathrm{M})$ less than the number of discontinuities are removed from the cavity's mode spectrum [3]. Finally, the shape of the cavity was tuned such that the $\mathrm{TM}_{0 \mathrm{~N} 0}$ modes are harmonic. This was accomplished by iteratively modifying the cavity's geometry and solving for the first $20 \mathrm{TM}_{\mathrm{ON} 0}$ mode frequencies with the field solver Poisson Superfish [4]. In the future, this manual evaluation of the Superfish solutions could be replaced with an automated computer-based search algorithm that would likely provide a more finely tuned geometry based on stricter resonance criteria for each mode.

Superfish is a field solver designed for cylindrically symmetric cavities. As such, the slits were not incorporated into the Superfish file; however, the presence of the slits on the actual cavity did not appear to adversely affect the accuracy of the Superfish result. The graphical field solutions of modes $\mathrm{TM}_{010}$ through $\mathrm{TM}_{080}$ are shown in Fig. 1, with the cavity shown in cross section and the beam axis horizontal.

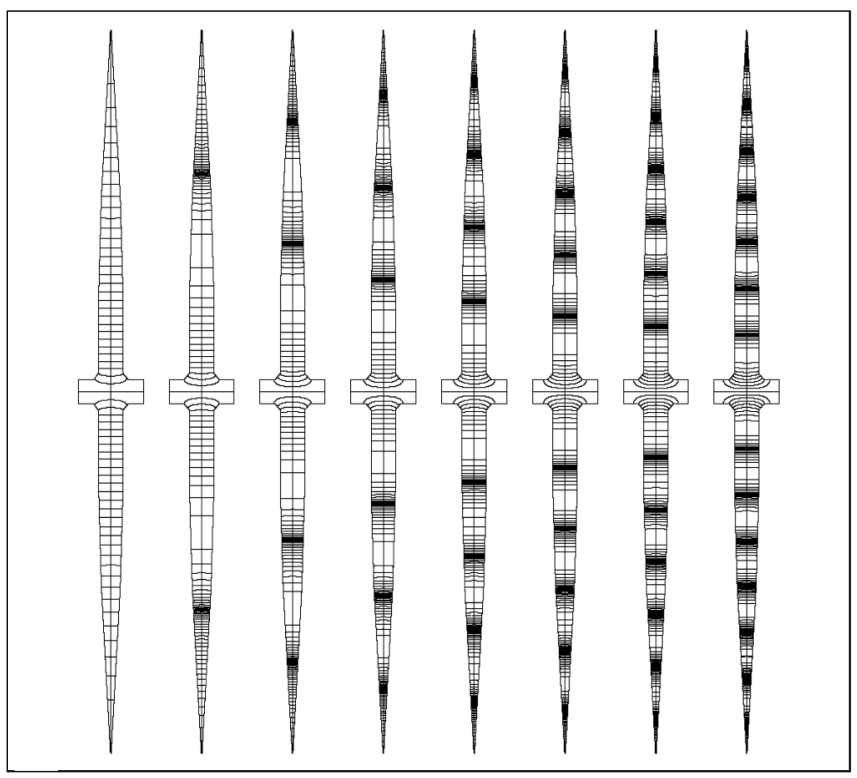

FIG. 1. The TM modes of the cavity bunch-length monitor from $\mathrm{TM}_{010}$ through $\mathrm{TM}_{080}$.
The final Superfish solution was programmed into a computer numerically controlled milling machine. Cavity halves were manufactured from $12.5 \mathrm{~mm}$ thick aluminum sheet, cut into the shape of disks with outer diameter $\sim 20 \mathrm{~cm}$ and with an $8 \mathrm{~mm}$ diameter beam line aperture drilled through the center of each disk. Material was removed to form the interior cavity surface and then 48 radial slots were cut into the back side of each cavity half using a $3 \mathrm{~mm}$ diameter ball-shaped end mill, with a depth that contoured the cavity but did not penetrate it. A thin slit was then milled into the bottom of each wide slit using a $0.8 \mathrm{~mm}$ diameter end mill that penetrated the cavity surface, creating electrical discontinuities, and improving the cavity vacuum conductance.

Two fully functional bunch-length monitors were constructed, with different methods employed to mate the two cavity halves. For one bunch-length monitor, the cavity halves were welded together and, for the other, the two cavity halves were bolted together with an intervening wire seal. Once the cavity halves were attached, the structure was then bolted to the interior of a 13.25-inch diameter double-sided knife-edge ConFlat flange using three aluminum support tabs. An ultrahigh vacuum SMA feedthrough was welded to the outside edge of the double-sided knifeedge ConFlat flange. A $50 \mathrm{Ohm}$ taper reduces the feedthrough's coaxial diameter to $3 \mathrm{~mm}$ and accommodated a $1 \mathrm{~mm}$ diameter rigid copper center conductor. The coaxial feedthrough penetrates the cavity through its plane of symmetry and the copper center conductor continues along this plane toward the edge of the beam aperture where it loops to the ground forming a broadband magnetic field coupler. The cavity and double-sided knife-edge flange where then sandwiched between 13.25-inch to 2.75-inch reducing flanges, to form the vacuum enclosure. Figure 2 shows different views of the cavity bunch-length monitor during construction.

Both bunch-length monitors were evaluated using a network analyzer and a dipole antenna placed on-axis as a surrogate for the beam. Measurements (Table I) for both cavities were very similar and confirmed that the cavity mode spectrum was nearly harmonic and with a bandwidth of approximately $19 \mathrm{GHz}$, supporting twelve modes $\left(\mathrm{TM}_{0,1,0}-\mathrm{TM}_{0,12,0}\right)$. Low $\mathrm{Q}$ values were expected due to the high surface area to volume ratio of the cavity geometry, and due to the relatively high surface resistance of aluminum. The first two modes of both cavities exhibited the largest deviation from desired resonant frequency (fundamental frequency $1497 \mathrm{MHz}$ ) and the weakest S21 coupling which describes the coupling efficiency from the radiating dipole to the cavity antenna and electrical feedthrough. The bunch-length monitor that employed the bolted-together cavity halves operated closer to the desired resonant frequency because slight adjustments to cavity dimensions could be made by applying different levels of pressure to the intervening wire seal. The bunch-length 

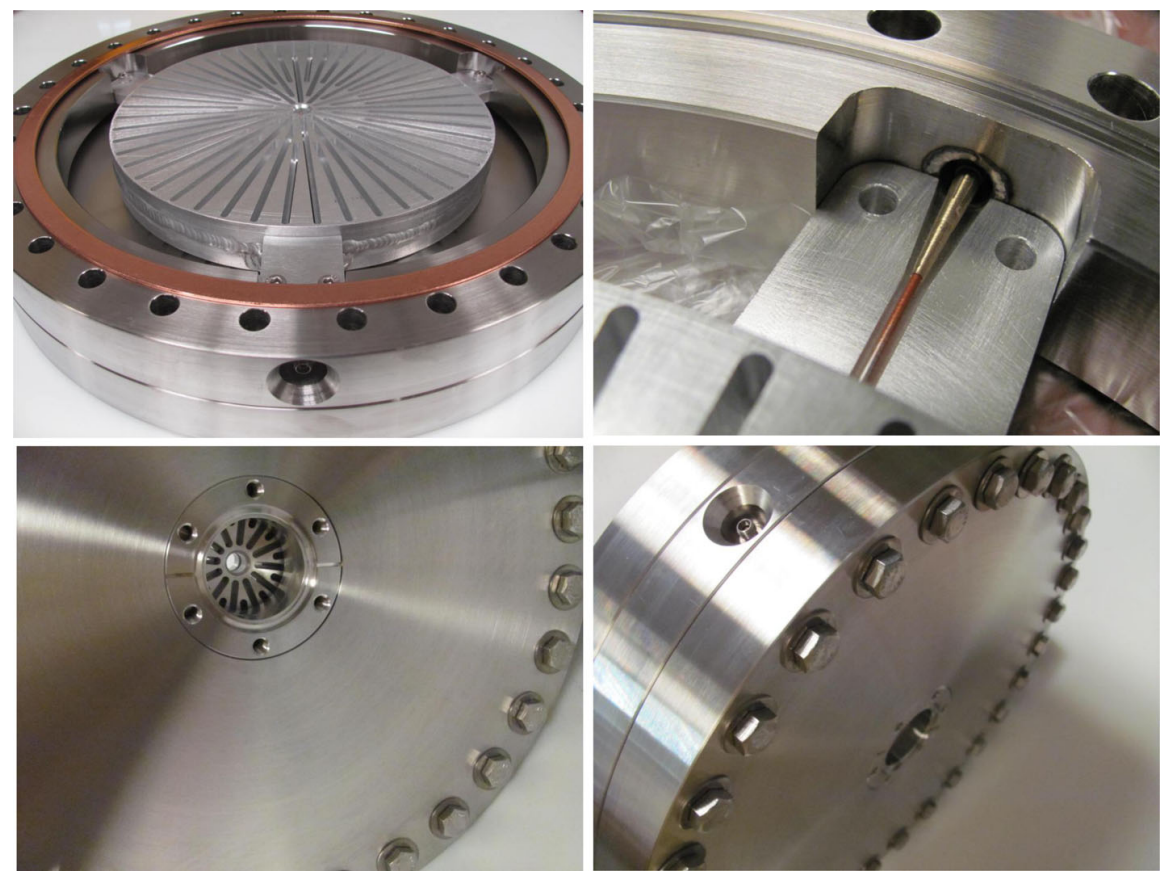

FIG. 2. The cavity bunch-length monitor during construction.

monitor with welded cavity halves was sent to Jefferson Lab for beam-based evaluation.

\section{B. Photogun and diagnostic beam line}

Experiments were performed using an ultrahigh vacuum load-locked DC high voltage photogun $[5,6]$ and diagnostic beam line described in detail in Ref. [7] (Fig. 3). The load-locked gun consists of a photocathode preparation chamber and high voltage chamber, separated by a bakable all-metal gate valve. Photoemission from GaAs requires an atomically clean surface with a work function that lies below the conduction band level [8]. This was achieved by heating the photocathode to $\sim 500^{\circ} \mathrm{C}$ inside the preparation chamber to remove weakly bound adsorbed gas. The photocathode was then cooled to room temperature and approximately one monolayer of cesium and nitrogen trifluoride was applied to the surface. Following this procedure, the photocathode was moved into the cathode electrode inside the high voltage chamber, where it was illuminated with laser light to generate an electron beam.

The photogun could be operated over a wide range of bias voltages up to $200 \mathrm{kV}$, and photocathodes could be quickly replaced. For this experiment, two photocathodes were used: a strained-superlattice GaAs-GaAsP

TABLE I. Bench test measurements for the bunch-length monitor with bolted-together cavity halves and intervening wire seal. Similar results were obtained for the welded bunch-length monitor used for beam-based measurements.

\begin{tabular}{lcccc}
\hline \hline $\begin{array}{l}\mathrm{TM}_{\text {ONo mode }} \text { m } \\
\text { number N }\end{array}$ & $\begin{array}{c}\text { Frequency } \\
(\mathrm{MHz})\end{array}$ & $\begin{array}{c}\text { Frequency/N } \\
(\mathrm{MHz})\end{array}$ & Measured Q & $\begin{array}{c}\text { S21 Coupling to } \\
\text { on-axis dipole (dB) }\end{array}$ \\
\hline 1 & 1550 & 1550 & 159 & -25 \\
2 & 3031 & 1515 & 108 & -18 \\
3 & 4493 & 1497 & 155 & -12 \\
4 & 5953 & 1488 & 212 & -10 \\
5 & 7445 & 1489 & 165 & -11 \\
6 & 8952 & 1492 & 325 & -11 \\
7 & 10417 & 1488 & 520 & -12 \\
8 & 11885 & 1486 & 490 & -10 \\
9 & 13403 & 1489 & 382 & -10 \\
10 & 14893 & 1489 & 867 & -9 \\
11 & 16386 & 1489 & 936 & -11 \\
12 & 17880 & 1490 & 993 & -12 \\
\hline \hline
\end{tabular}




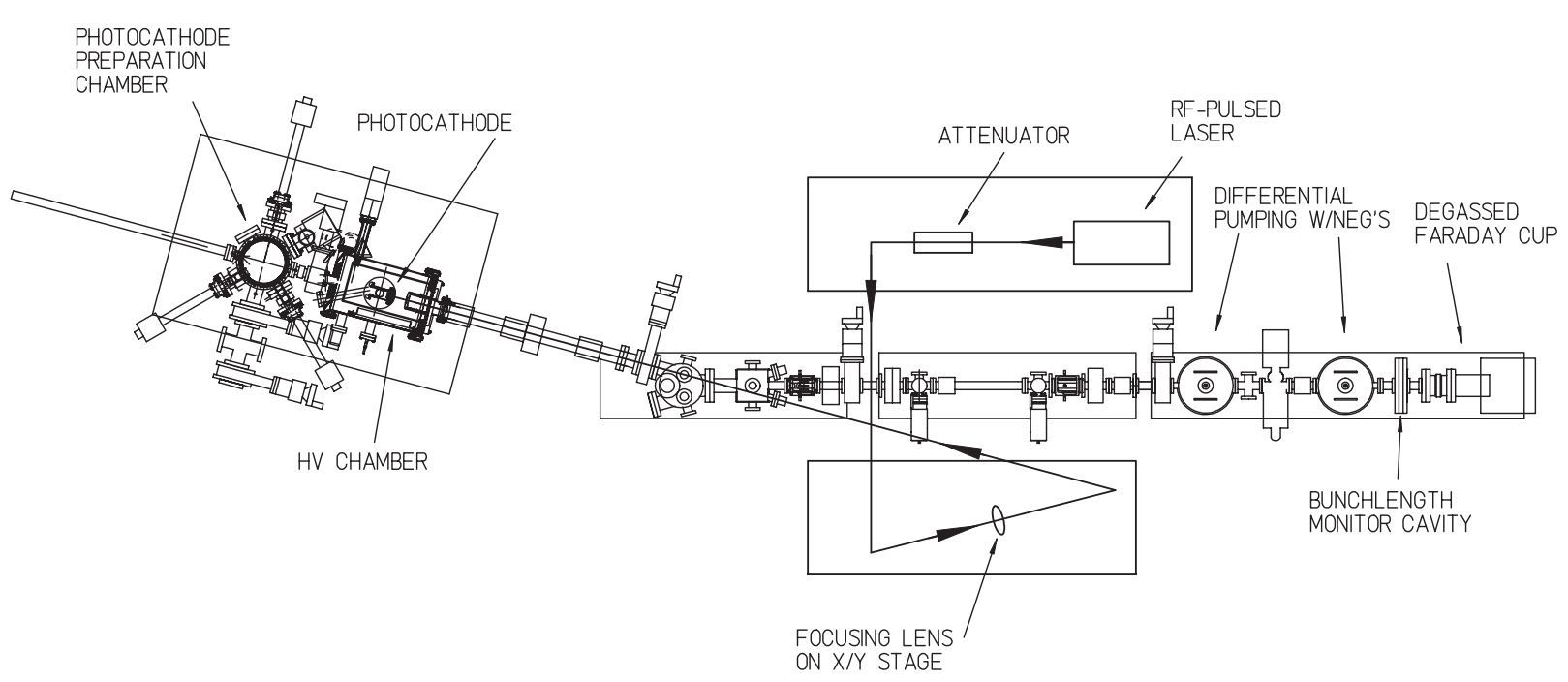

FIG. 3. The DC high voltage load-locked GaAs photogun and diagnostic beam line. The cavity bunch-length monitor was positioned near the beam dump. Besides the labeled components, the beam line consisted of valves, vacuum pumps, view screens, beam position monitors, a wire scanner, steering and focusing magnets.

photocathode with $100 \mathrm{~nm}$ thick active layer [9], and a thick $(625 \mu \mathrm{m})$ unstrained bulk GaAs photocathode [10].

Photoelectrons passed through a hole in the anode and were delivered to a water-cooled Faraday cup beam dump $5 \mathrm{~m}$ away. Solenoid magnets focused the beam and weak steering magnets kept the beam centered in the beam pipe. The 15 degree bend allowed illumination of the photocathode at normal incidence without needing mirrors inside the vacuum chamber. The field strength of the magnets was adjusted for each photogun bias voltage.

The cavity bunch-length monitor was located near the Faraday cup beam dump. Immediately following installation, the cavity and adjoining beam line were baked under vacuum at $250^{\circ} \mathrm{C}$ for 30 hours. At the end of the bake cycle, the nonevaporable getter (NEG) pumps inside an adjacent differential pump station were heated to $400^{\circ} \mathrm{C}$ for 20 minutes to maximize the NEG pump speed. Although no pressure gauges were available in this section of the beam line, the vacuum level was deemed very good $\left(10^{-11}\right.$ Torr or better) based on measured current of nearby ion pumps. The photogun vacuum level did not change when the valve to the bunch-length cavity and beam dump was opened.

The drive laser consisted of a low-power gain-switched fiber-coupled diode laser operating at $1560 \mathrm{~nm}$ followed by a $10 \mathrm{~W}$ commercial Er:Yb fiber amplifier [11]. Useful light at $780 \mathrm{~nm}$ was obtained via second harmonic generation inside a periodically poled lithium niobate crystal to obtain approximately $2 \mathrm{~W}$ average power. Laser pulse width measurements using a fast photodiode indicated 36.6 ps pulses (FWHM), consistent with optical autocorrelator measurements made previously [11]. The pulse repetition rate could be set to any frequency over a broad range by simply adjusting the frequency of the applied rf to the gain-switched diode laser. The laser beam was focused to $500 \mu \mathrm{m}$ diameter (Gaussian FWHM) at the photocathode.

The bunch-length monitor cavity was attached to a $40 \mathrm{GHz}$ sampling head (Tektronix SD-30) using a 3/8inch diameter Heliax cable so that the oscilloscope (Tektronix 11801b) could to be operated from behind an $\mathrm{x}$-ray shield wall. To ensure the cable did not significantly contribute dispersion to the measurement, the cavity response was characterized using different cable lengths, from 2 to $12 \mathrm{~m}$. The shortest cables provided the most signal but the shape of the oscilloscope trace was independent of cable length, indicating that cable dispersion was not a significant factor.

\section{RESULTS}

The first measurements were made using electron beam delivered from the strained-layer GaAs/GaAsP superlattice photocathode with $100 \mathrm{~nm}$ thick active layer. The oscilloscope signal in Fig. 4(a) shows the laser optical pulse train obtained using a photodiode and Fig. 4(b) shows the output from the cavity bunch-length monitor when the photogun was biased at $175 \mathrm{kV}$ and with $500 \mu \mathrm{A}$ delivered to the beam dump. The pulse repetition rate of the drive laser was varied to maximize the peak-to-peak signal of the oscilloscope: a repetition rate of $1533 \mathrm{MHz}$ provided the most signal and the most narrow pulse shape. The repetition rate could be varied $\pm 20 \mathrm{MHz}$ before the signal fell by a factor of 2 . These findings were consistent with bench testing results.

The cavity antenna couples to the magnetic field of the cavity modes which is phase delayed by 90 degrees from the electric field resulting in an oscilloscope signal [Fig. 4(b)] with positive and negative peaks. To obtain the electric field versus time waveform, the cavity signal was 

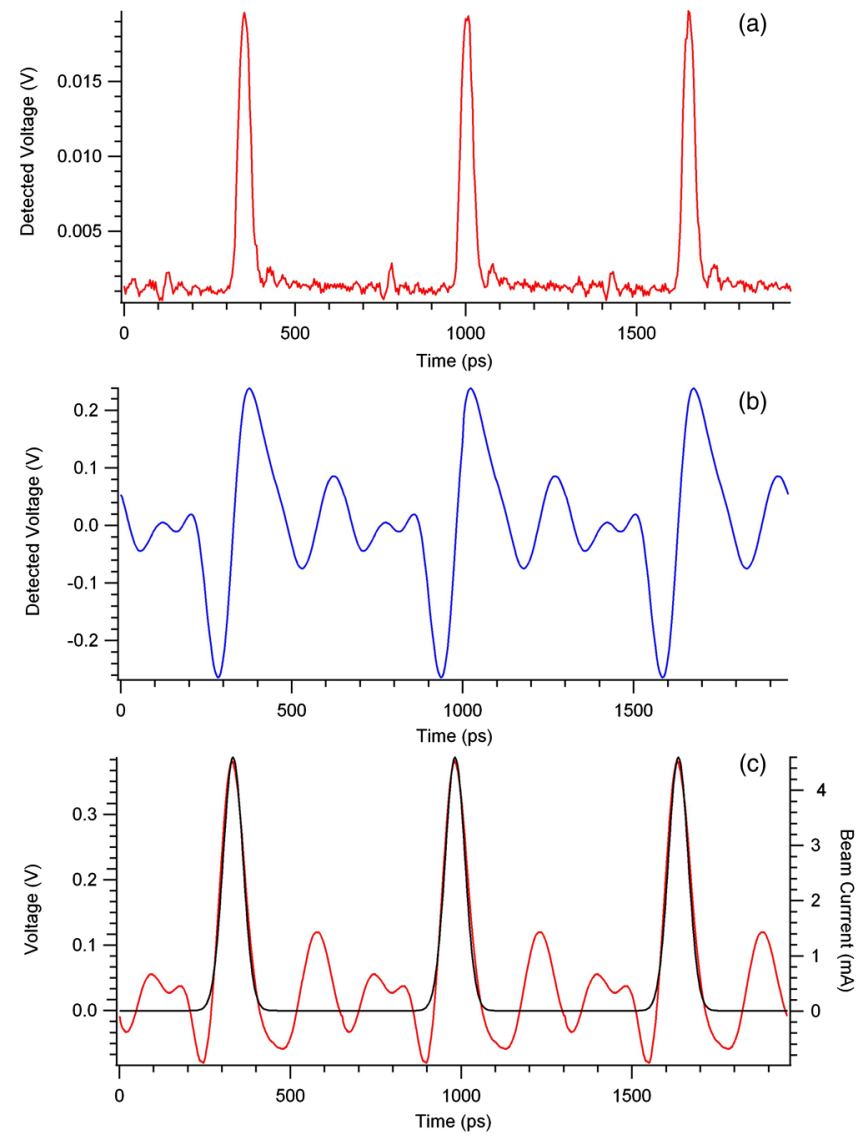

FIG. 4. Oscilloscope traces of (a) the drive laser optical pulse train, (b) the cavity bunch-length monitor, and (c) the FFT of signal from the cavity bunch-length monitor together with an overlaid Gaussian fit.

postprocessed by applying a fast Fourier transform (FFT) using the software program IGOR [12], yielding a complex output. In the complex plane, multiplication by $i$ results in a 90-degree phase rotation. The complex output of the waveform's FFT was then multiplied by $-i$ and its inverse FFT was computed yielding a pulse train that reflects the Gaussian shape of the laser pulse [Fig. 4(c)].

The "ringing" clearly visible along the baseline of the pulse is caused by the cavity's limited bandwidth and the nonuniform response at each mode. A perfectly tuned cavity with infinite bandwidth would produce a signal with a flat baseline, representative of the Gaussian fit applied to the trace of Fig. 4(c). However, despite the limited bandwidth and uncertainty in cavity response at each mode, the fit to the dominant portion of the signal is good. No attempt was made to correct the postprocessed result for limited cavity bandwidth or nonuniform response. The postprocessed result shown in Fig. 4(c) indicates the electron bunch length was $67 \mathrm{ps}$ (FWHM) and the peak current of the bunch was $4.6 \mathrm{~mA}$, as calculated using the expression below, where the denominator is the area of the Gaussian fit multiplied by the bunch rate:

$$
I_{\text {peak }}=\frac{I_{\text {average }}}{f \sigma \sqrt{2 \pi}}
$$

\section{A. Results: Space charge induced bunch-length growth}

Space-charge induced bunch-length broadening is a common problem for DC high voltage photoguns, even at $\mathrm{pC}$ bunch charge. Bunch-length broadening is most problematic at low bias voltages and/or higher current and bunch charge. The cavity bunch-length monitor was used to characterize bunch-length broadening as a function of bias voltage and beam current as shown in Fig. 5(a), which shows the spacing between the positive and negative peaks of the raw oscilloscope data. The FWHM bunch length is approximately equal to $80 \%$ of the positive and negative peak spacing, an empirically determined relationship. Operation at the highest bias voltage $(195 \mathrm{kV})$ produced the stiffest beam: the positive-negative peak spacing increased from a minimum detectable value of $\sim 76$ to $86 \mathrm{ps}$ when the average current was varied from 25 to $500 \mu \mathrm{A}$, corresponding to a bunch charge variation of 0.015 to $0.3 \mathrm{pC}$. In stark contrast, the peak spacing increased to over $250 \mathrm{ps}$ over the same current and bunch charge range when the bias voltage was reduced to $75 \mathrm{kV}$.

The minimum detectable positive/negative peak spacing was $76 \mathrm{ps}$, corresponding to a $57 \mathrm{ps}$ bunch length
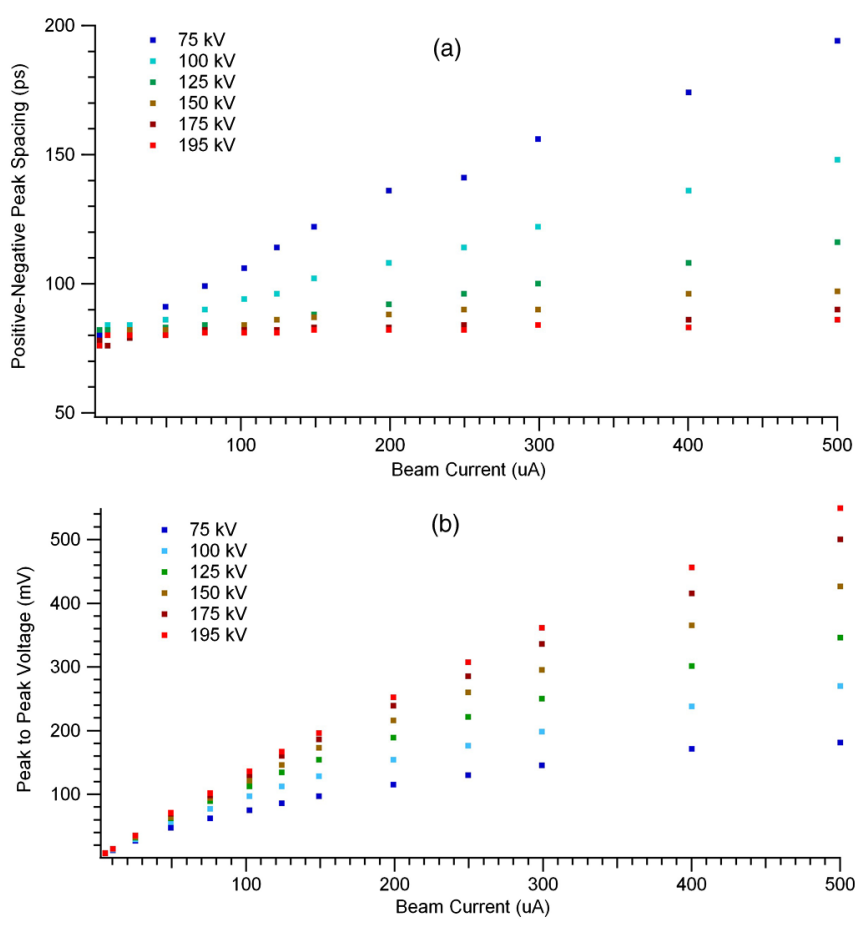

FIG. 5. (a) The spacing of the positive and negative peaks as a function of beam current and photogun bias voltage. (b) The sensitivity of the cavity bunch-length monitor as a function of average beam current and photogun bias voltage. 
(FWHM) when the Gaussian fit was applied to the postprocessed waveform. This minimum value was observed at low average beam current where space charge forces are small, for all of the tested bias voltages. It is reasonable to assume the actual bunch length at low current was smaller than measured, and closer to the laser optical pulse width of 36.6 ps. A cavity with more bandwidth would resolve narrower bunches.

The cavity-signal peak-to-peak voltage is plotted in Fig. 5(b), as a function of gun voltage and beam current. The slope of the line defines the cavity sensitivity. At low current and bunch charge, where space charge forces are relatively benign, the cavity sensitivity is independent of gun bias voltage, corresponding to $\sim 100 \mathrm{~V} / \mathrm{amp}$, when comparing peak detected voltage to peak current in the bunch. As the bunch length grows, particularly for low bias voltage operation, the cavity sensitivity is greatly diminished. This is partially attributed to weaker coupling of the first few cavity modes. Bunches with significant space charge growth have large amplitude coefficients in the first few terms of their Fourier series expansion that correspond to cavity modes that are less well coupled.

Figure 6 shows cavity oscilloscope traces (left side) at low and high bias voltage (75 and $175 \mathrm{kV}$ ), for beam currents 25, 250, and $500 \mu \mathrm{A}$. The postprocessed traces are shown of the right side of Fig. 6. The images in Fig. 6 indicate prominent bunch-length broadening at low bias voltage, and more interestingly, the images in Fig. 6(b) indicate the shape of the bunch is significantly altered due to space charge forces, even becoming double peaked. The cavity bunch-length monitor clearly distinguishes bunches
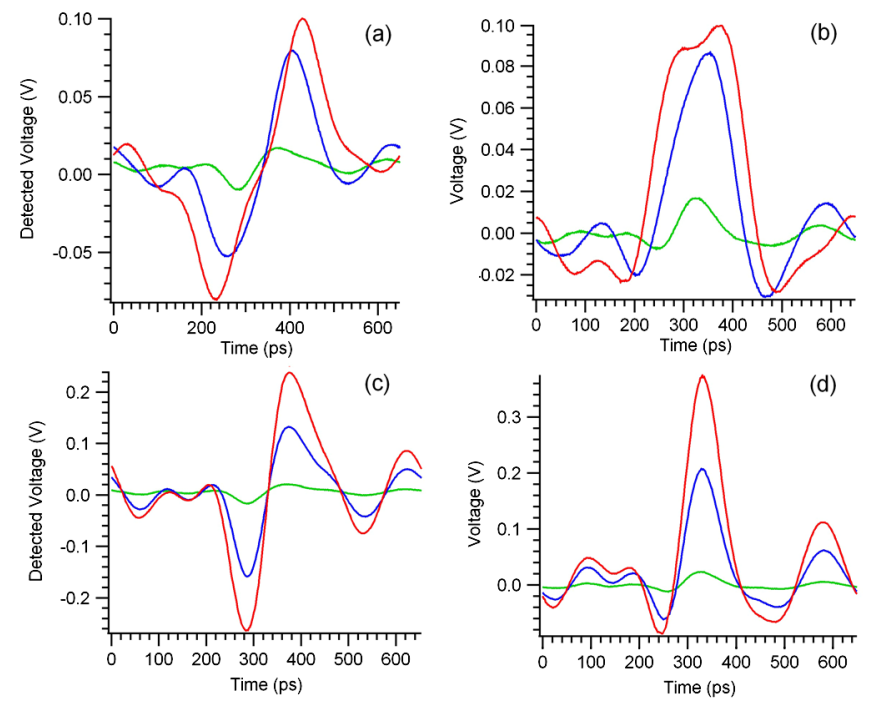

FIG. 6. Electron bunches from thin $(100 \mathrm{~nm})$ strained-layer superlattice GaAs/GaAsP photocathodes. The left side images show oscilloscope traces and the right side images show postprocessed FFTs, for $75 \mathrm{kV}$ bias voltage (b) and $175 \mathrm{kV}$ bias voltage (d) at three currents. produced with different space charge induced temporal aberration.

\section{B. Results: Comparing bunches from thin and thick GaAs}

The thin GaAs/GaAsP strained-superlattice photocathode was replaced with thick $(625 \mu \mathrm{m})$ bulk GaAs, which is a common photocathode material used for accelerator light-source applications [13]. Light travels relatively deep into the GaAs material before being completely absorbed $(>1 \mu \mathrm{m})$, particularly light at $780 \mathrm{~nm}$ [14]. The photoexcited electrons that originate deep within the material take longer to reach the surface. As a result, the electron bunches that are produced from thick GaAs exhibit relatively long tails compared to bunches produced from thin material [15]. Bunch traces from the thick and thin GaAs photocathodes are plotted in Fig. 7. The cavity bunchlength monitor clearly resolves the electron tail associated with thick GaAs, at low and high beam currents, even though the bunch length from both photocathodes is comparable.
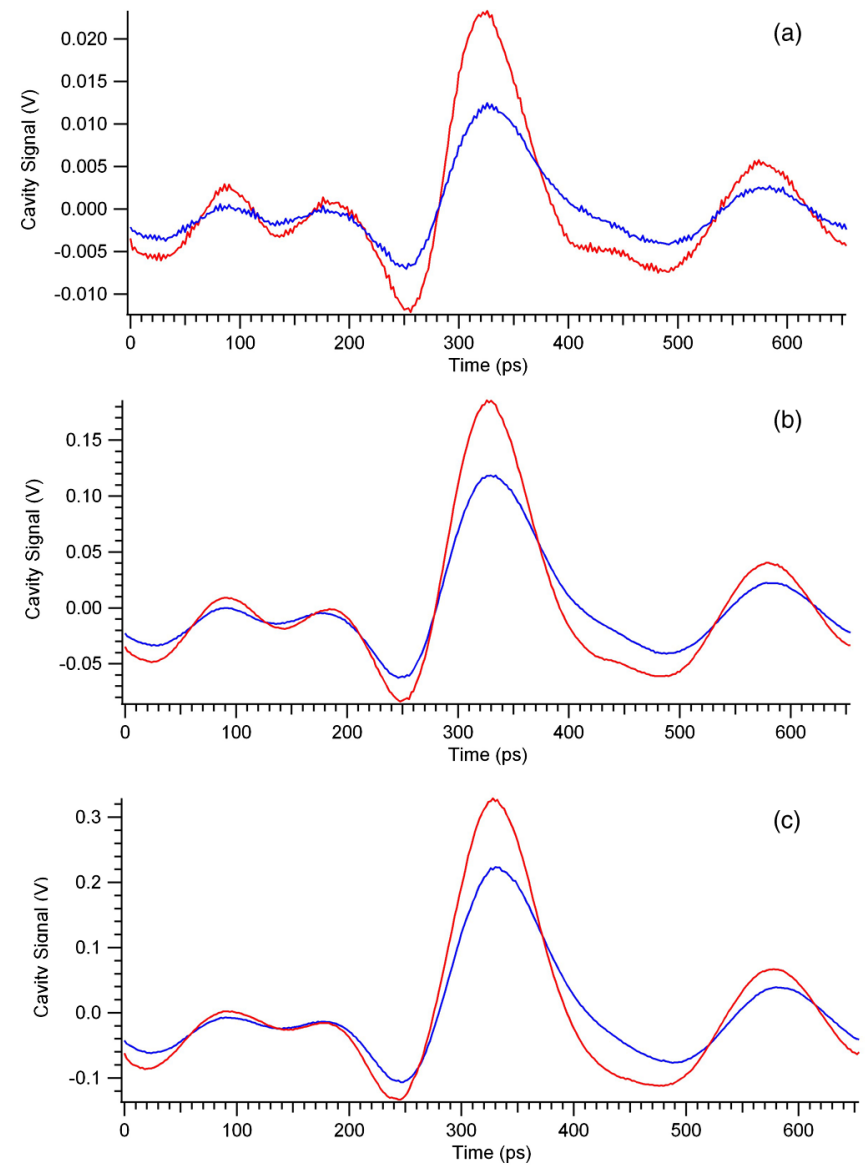

FIG. 7. Comparison of bunches from thick GaAs (blue traces) and thin strained-layer superlattice $\mathrm{GaAs} / \mathrm{GaAsP}$ photocathodes (red traces) at $175 \mathrm{kV}$ bias voltage and at three currents: (a) $25 \mu \mathrm{A}$, (b) $250 \mu \mathrm{A}$, and (c) $500 \mu \mathrm{A}$. 


\section{CONCLUSION}

A harmonically resonant cavity was constructed that produces a real-time signal that is representative of the Fourier series expansion of the repetitive bunched beam passing through its bore. The cavity exhibited bandwidth capable of resolving $57 \mathrm{ps}$ bunches (FWHM) and with sensitivity as high as $100 \mathrm{~V} / \mathrm{amp}$ (peak current), when attached directly to an oscilloscope. The cavity could clearly detect space charge induced bunch lengthening, and different tail signatures associated with beam production from different photocathode materials.

Benchtop rf measurements indicated the twelve resonant modes were nearly harmonic and the cavity spectral response was relatively flat across the entire spectrum; however, future cavity construction could be improved using an automated optimization algorithm to ensure strict resonance criteria and uniform coupling across the entire spectrum. In addition, a more detailed beam-based quantitative assessment of cavity performance awaits cross comparison with a calibrated bunch-length monitor, such as the rfdeflecting cavity "chopper" at the Continuous Electron Beam Accelerator Facility at Jefferson Lab. In addition, a better commercial electrical feedthrough with $20 \mathrm{GHz}$ rated bandwidth should provide improved resolution, enabling the detection of shorter bunches.

This demonstration lends credibility to the idea that these cavities could be used in other applications, including bunching and shaping, when driven with external rf. For example, more sophisticated bunch shaping could be accomplished by selectively adding or subtracting to the individual terms of the beam's Fourier series expansion. In this manner it would be possible to correct temporal aberration, or to produce arbitrary bunch shapes. These tasks cannot be accomplished using traditional brute-force bunching using a single-mode cavity.

\section{ACKNOWLEDGMENTS}

This paper was authored in part by Jefferson Science Associates under U.S. DOE Contract No. DE-AC0584ER40150. The authors are indebted to Professor Jim Ellison, Professor Edl Schamiloglu, and Dr. Klause Heinemann of the University of New Mexico and to Curt Hovater and Mark Wiseman of Jefferson Lab, for discussions, measurements, and helpful advice. This work was also previously funded by U.S. DOE SBIR Phase I Award No. DESC000266.

[1] R. Webber, Longitundinal Emittance: An Introduction to the Concept and Survey of Measurement Techniques
Including Design of a Wall Current Monitor, AIP Conf. Proc. No. 212 (AIP, New York, 1889), pp. 85-126.

[2] C. K. Sinclair, M. Poelker, P. A. Adderley, B. M. Dunham, J. C. Hansknecht, P. Hartmann, J. S. Price, P. M. Rutt, W. J. Schneider, and M. Steigerwald, Phys. Rev. ST Accel. Beams 10, 023501 (2007).

[3] C.A. Balanis, Advanced Engineering Electromagnetics (John Wiley and Sons, Inc., New York, 1989).

[4] K. Halbach and R.F. Holsinger, Part. Accel. 7, 213 (1976).

[5] P. A. Adderley, J. Clark, J. Grames, J. Hansknecht, K. Surles-Law, D. Machie, M. Poelker, M. L. Stutzman, and R. Suleiman, Phys. Rev. ST Accel. Beams 13, 010101 (2010).

[6] J. M. Grames, P. A. Adderley, J. Clark, J. Hansknecht, M. Poelker, M. L. Stutzman, R. Suleiman, K. Surles-Law, M. BastaniNejad, and J.L. McCarter, in Proceedings of the 2011 Particle Accelerator Conference, NY, USA (IEEE, New York, 2011).

[7] J. Grames, R. Suleiman, P. A. Adderley, J. Clark, J. Hansknecht, D. Machie, M. Poelker, and M.L. Stutzman, Phys. Rev. ST Accel. Beams 14, 043501 (2011).

[8] D. T. Pierce, F. Meier, and P. Zürcher, Appl. Phys. Lett. 26, 670 (1975); D. T. Pierce and F. Meier, Phys. Rev. B 13, 5484 (1976).

[9] T. Maruyama, D.-A. Luh, A. Brachmann, J. E. Clendenin, E. L. Garwin, S. Harvey, J. Jian, R. E. Kirby, C. Y. Prescott, R. Prepost, and A. M. Moy, Appl. Phys. Lett. 85, 2640 (2004).

[10] Bulk GaAs purchased from AXT Inc., 4281 Technology Drive, Fremont, CA 94538 [http://www.axt.com/]. Specifications: vertical gradient freeze, GaAs single crystal prime, Epi Ready, surface with 100 cleave plane orientation, $\mathrm{Zn}$ dopant at 5e18 carrier concentration/ $\mathrm{cm} 3, \sim 5000$ etch pit density.

[11] J. Hansknecht and M. Poelker, Phys. Rev. ST Accel. Beams 9, 063501 (2006).

[12] Igor Pro Version 6.2, Wavemetrics Inc., Lake Oswego, OR 97035 http://www.wavemetrics.com

[13] C. K. Sinclair, ICFA Newsletter 46, p. 97 (2008); Nucl. Instrum. Methods Phys. Res., Sect. A 557, 69 (2006).

[14] S.M. Sze, Physics of Semiconductor Devices (Wiley, New York, 1981), 2nd ed.

[15] P. Hartmann, Ph.D. dissertation, Shaker Verlag, Aachen, Germany, 1998; P. Hartmann, J. Bermuth, J. Hoffmann, S. Kobis, E. Reichert, H. G. Andresen, K. Aulenbacher, P. Drescher, H. Euteneuer, H. Fischer, P. Jennewein, K.-H. Kaiser, H. J. Kreidel, Ch. Nachtigall, S. Plutzer, M. Schemies, K.-H. Steffens, M. Steigerwald, H. Trautner, D. v. Harrach, I. Altarev, R. Geiges, K. Grimm, Th. Hammel, E. Heinen-Konschak, H. Hofmann, E.-M. Kabuss, A. Lopes-Ginja, F.E. Maas, and E. Schilling, Nucl. Instrum. Methods Phys. Res., Sect. A 379, 15 (1996). 